\title{
Simulating Inelastic Scattering in Scanning Transmission Electron Microscopy using $\mu$ STEM
}

\author{
A.J. D’Alfonso ${ }^{1}$, S.D. Findlay ${ }^{2}$ and L.J. Allen ${ }^{1}$ \\ 1. School of Physics, University of Melbourne, Parkville, Victoria 3010, Australia \\ 2. School of Physics and Astronomy, Monash University, Clayton, Victoria 3800, Australia
}

The ability to image specimens of condensed matter at atomic resolution has vastly expanded our understanding of advanced materials and their properties. However, as electron scattering is a highly complicated process much attention has been directed toward developing our understanding of imaging formation and interpretation. The simultaneous measurement of high angle annular dark field and electron energy loss spectroscopy images has been one approach toward improved interpretation [1], especially if chemical specificity is desired [2]. However, even with multimode imaging, it is widely acknowledged that in order to extract the maximal information present in atomic resolution images, or possibly to reveal potential ambiguities in image appearance, accurate simulations of the imaging process are critical [3].

We will discuss the application of image simulation to materials design problems, emphasizing the importance of using sophisticated simulations and models, coupled with careful experiment, to place electron microscopy on an absolute scale. One microscopy simulation suite, which is freely available for download, capable of simulating images based on elastic and inelastic phonon scattering, inner-shell ionization and subsequent x-ray generation is $\mu$ STEM [4]. The $\mu$ STEM suite is based on the multislice algorithm implemented using fast Fourier transforms. The advantages of this approach are that it is computationally efficient and lends itself naturally to parallelization. $\mu$ STEM is available for both GPU and CPU computing models which allows detailed simulations to be performed rapidly on a desktop computer. Figure 1, taken from Ref. [5] shows a selection of imaging modes that may be simulated using $\mu$ STEM, including those based on inner-shell ionization.

$\mathrm{STEM}$ is not limited to simulating periodic specimens, like that indicated in Fig. 1. As an example, consider the situation depicted in Figure 2(a) of a cerium dioxide nanoparticle illuminated by a defocussed STEM probe. Figure 2(b) shows an experimentally recorded Ronchigram (or Gabor Hologram) while Figure 2(c) shows the corresponding simulation. The experimentally recorded Ronchigram was subsequently used to reconstruct the phase image of the complex specimen transmission function, Fig. 2(d). Confirmation that the reconstruction process gave a unique solution was verified through forward simulation, Fig. 2(e). In this case, combining simulation and experiment was critical to confirm the experimental reconstruction.

[1].D.A. Muller, et al, Nature, 399, (1999), 758.

[2].P. Wang, et al, Phys. Rev. Lett. 101, (2008), 236102.

[3].C.L. Jia, et al. Nature materials, 13, (2014), 1044-1049.

[4].http://tcmp.ph.unimelb.edu.au/mustem/muSTEM.html

[5].L.J. Allen, A.J. D’Alfonso and S.D. Findlay. Ultramicroscopy, in press, (2014).

[6].This research was supported under the Australian Research Councils Discovery Projects funding scheme (Projects DP110102228 and DP140102538) and its DECRA funding scheme (Project DE130100739). 

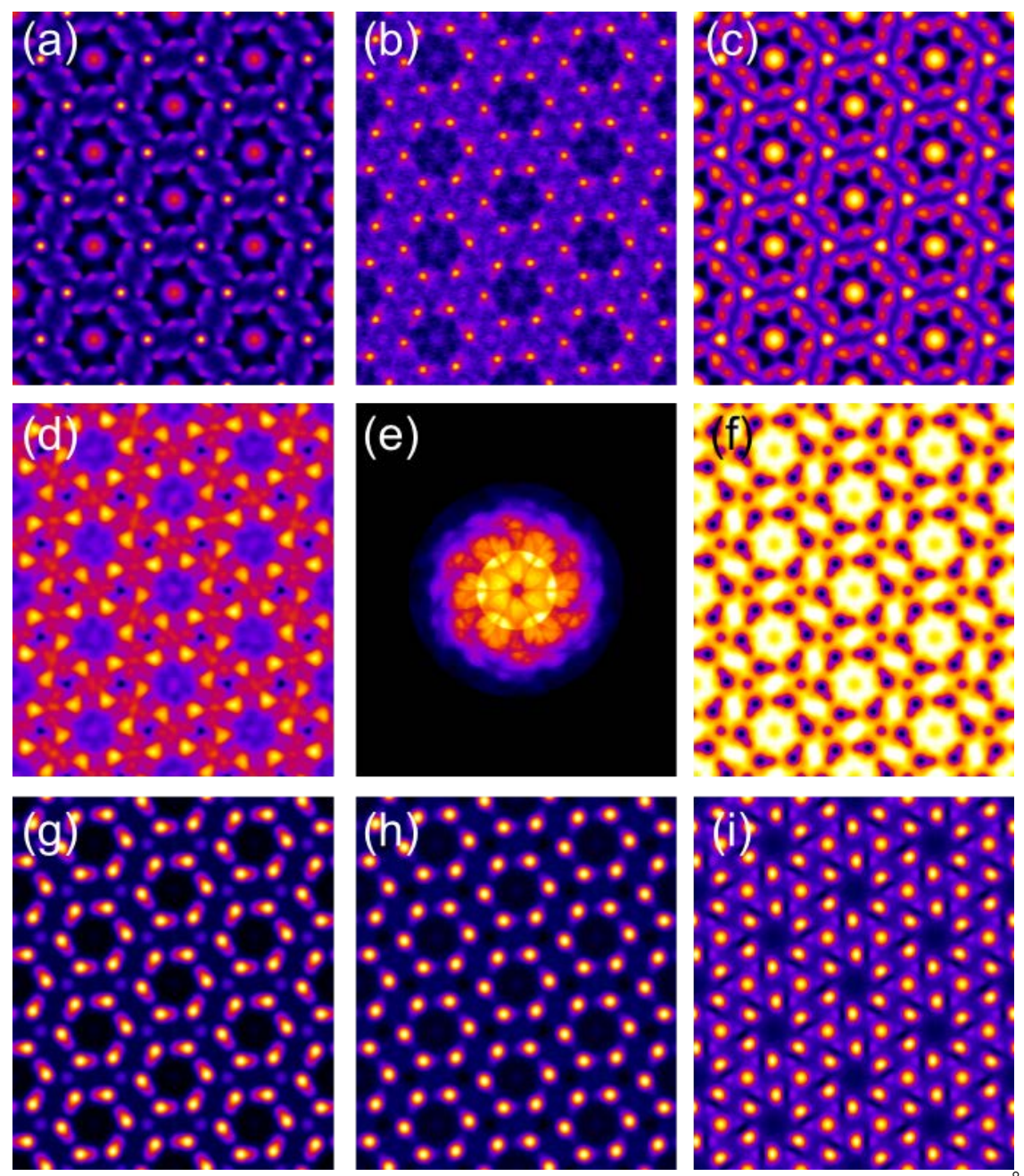

Figure 1 Images calculated using $\mu \mathrm{STEM}$ for $100 \mathrm{keV}$ electrons on a $300 \AA$ thick specimen of [0001] $\mathrm{Si}_{3} \mathrm{~N}_{4}$. (a) Exit surface intensity due to elastically scattered electrons and (b) exit surface intensity due to thermally scattered electrons for plane wave illumination. In (c) and (d) the intensities in parts (a) and (b) after imaging by an aberration free lens with an aperture of $25 \mathrm{mrad}$ are shown (e) A position averaged convergent beam electron diffraction pattern calculated using an aberration-free, coherent probe formed using an aperture of $9.6 \mathrm{mrad}$ with the average taken over the unitcell. (f) The annular bright-field image and (g) a high-angle annular dark-field image, using a probe formed using a 25 mrad aperture. Inner and outer angles are given in the text. An elemental map based on the energy-dispersive x-ray signal for the Si K edge is shown in (h) and for the N K edge in (i), using a probe forming aperture of 25mrad. Each image is displayed on its own contrast scale.
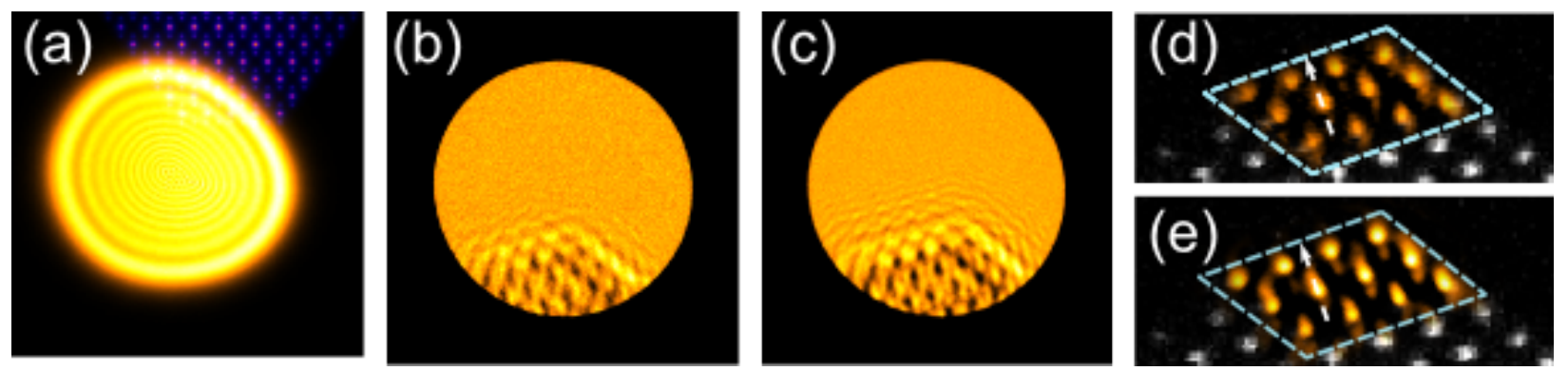

Figure 2 (a) Probe sample configuration, and the corresponding (b) experimental and (c) simulated Ronchigrams. Reconstructed phase image of the specimen transmission function from (d) experiment and (e) simulation. The incident $300 \mathrm{keV}$ electron probe was formed using an aperture of $24 \mathrm{mrad}$ and a defocus of -80 $\mathrm{nm}$. 